\title{
Conoscopic observation of director reorientation during Poiseuille flow of a nematic liquid crystal
}

\author{
C. J. Holmes, ${ }^{1, a)}$ S. L. Cornford, ${ }^{2}$ and J. R. Sambles ${ }^{1}$ \\ ${ }^{1}$ Electromagnetic Materials Group, School of Physics, University of Exeter, Exeter EX4 4QL, \\ United Kingdom \\ ${ }^{2}$ HP Labs Bristol, Long Down Avenue, Stoke Gifford, Bristol BS34 8QZ, United Kingdom
}

(Received 10 August 2009; accepted 29 September 2009; published online 30 October 2009)

\begin{abstract}
Director reorientation under pressure driven (Poiseuille) flow is observed conoscopically for the liquid crystal $5 \mathrm{CB}$ aligned at an azimuthal angle of $45^{\circ}$ to the direction of flow. A polyimide surface treatment (AL 1254) is used to promote planar homogeneous alignment and rubbed to produce an initial azimuthal alignment angle $\phi_{0}$. Conoscopic interference figure rotation is documented as a function of flow rate and compared to that produced from numerical models using Leslie-EricksenParodi theory. Model and data show excellent agreement. (C) 2009 American Institute of Physics. [doi:10.1063/1.3251792]
\end{abstract}

The viscodynamics of pressure induced (Poiseuille) flow in nematic liquid crystals are important in many technological applications. These range from the use of low molar mass liquid crystals in the manufacture of liquid crystal displays to liquid crystal polymer (LCP) injection molding used to spin textile fibers. ${ }^{1}$ Director orientation and flow channel dimensions for fabrication techniques such as LCP injection molding can often play an important role in the order parameter ${ }^{2}$ and the number of defects in finished products. Other studies have examined nematic flow alignment in samples with colloidal dispersions and the topological defects associated with them, as commonly found in foods, paint and drugs. ${ }^{3}$

To date, much work has been carried out in examining director reorientation and measurement of various viscoelastic properties of nematic liquid crystals under shear induced (Couette) flow ${ }^{4,5}$ including work involving the shearing of nematic liquid crystals under varying initial azimuthal $(\phi)$ alignment conditions. ${ }^{6,7}$ Such shear flow experiments regularly involve the flowing of a liquid crystal sample between two glass plates by way of dragging one plate relative to the other at a constant velocity, producing a linear velocity distribution across the depth of the cell. In the Poiseuille flow study presented here, a distinctly different flow distribution is observed (particularly at low rates), ${ }^{8}$ a parabolic distribution of flow speeds symmetrical about $d / 2$ [see Fig. 1(a)], characteristic of any classical pressure gradient flow regime. Such a symmetric flow speed distribution results in differing azimuthal ( $\phi$-rotation) and tilt ( $\theta$-rotation) [see Fig. 1(b)] distortions through the depth of the sample compared to that of the linear velocity distribution produced from shear flow.

The dynamics of anisotropic fluid flow are inherently complex. This is due to the need for consideration of both the translational and orientational order of the constituent molecules under flow. Liquid crystals undergoing flow are well described by the continuum theory proposed by that of Leslie $^{9}$ and Ericksen, ${ }^{10}$ with five independent (Parodi ${ }^{11}$ ) viscosity coefficients $\left(\alpha_{1} \ldots \alpha_{5}\right)$ determining director orientation under perturbation from an external source. Such a theory (for the ideal case of no boundary effects) proposes that flow aligning liquid crystals $\left(\alpha_{3} / \alpha_{2}>0\right)$ rotate to achieve a con-

\footnotetext{
${ }^{a)}$ Electronic mail: c.holmes@ex.ac.uk.
}

stant angle $\theta_{L}$ (Leslie angle) out of the shear plane, at which point viscous torques on the director vanish. The Leslie angle is given by

$$
\theta_{L}=\tan ^{-1} \sqrt{\alpha_{3} / \alpha_{2}}
$$

Typical values of the Leslie angle are a few degrees away from the flow direction. ${ }^{12}$ In the case of non flow aligning $\left(\alpha_{3} / \alpha_{2}<0\right)$ systems, a continuous tumbling of the director in the direction of flow is observed until a cascade of instabilities occurs, leading at very high shear rates to director turbulence. ${ }^{12}$ As in real systems, surface anchoring effects (producing splay and twist gradients through the depth of the cell) will affect the absolute amount of director distortion. As liquid crystal flow speeds (and director distortion associated with viscous torques) increases, the effect of the elastic surface anchoring condition is decreased to a point where the boundary effects become negligible, $E_{r} \gg 1$ where $E_{r}$ is termed the Ericksen number, defined by

$$
E_{r}=\frac{\alpha_{3}-\alpha_{2}}{K_{1}} d^{2} v,
$$

where $v$ is the velocity of the moving plate, $d$ is the cell thickness, $K_{1}$ is the Frank splay elastic constant, and $\gamma_{1}$ $=\alpha_{3}-\alpha_{2}$ is known as the coefficient of nematic liquid crystal rotational viscosity or Tsvetkov's viscosity coefficient. ${ }^{13}$ Only when viscous torques negate the elastic anchoring forces can Eq. (1) be relied upon.

Due to the high level of birefringence exhibited by an ordered nematic liquid crystal, conoscopy (Fig. 2) enables measurement of both the average azimuthal director angle $\phi$ (a)
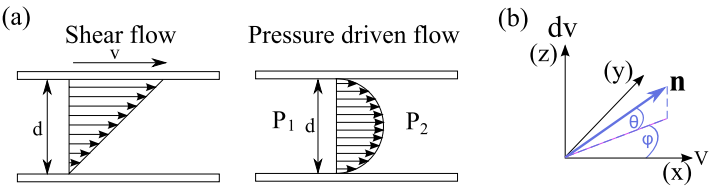

FIG. 1. (Color online) (a) A schematic diagram representing the difference in velocity distribution of shear flow (left image) and pressure driven flow (right image). (b) Definition of two polar angles $(\phi, \theta)$ that define director orientation. A rotation in the shear plane is characterized by an azimuthal $\phi$ (twist), and a rotation out of the shear plane denoted by a zenithal $\theta$ (tilt). 


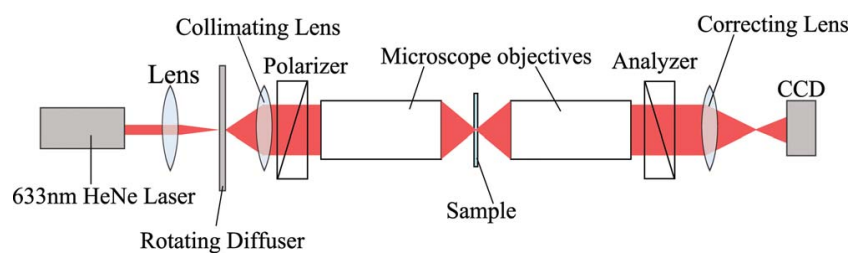

FIG. 2. (Color online) Schematic diagram of laser conoscope (see Ref. 15).

and the mean absolute tilt angle $|\bar{\theta}|$ exhibited by a sample. Viewing a birefringent sample with highly convergent monochromatic light through crossed polarizers allows characteristic interference figures to be examined ${ }^{14}$ where the conoscopic figure provides an optical average of the director orientation across the thickness of the entire cell. The distinct advantage is that azimuthal distortion is revealed by figure symmetry without accounting for the effect of tilt distortion on total birefringence. As in flow experiments, the director profile will be graduated from the top plate to the bottom plate due to wall effects at the surface and a distortion toward the Leslie angle in the bulk of the sample. ${ }^{7}$ As such, the conoscopic interference figure gives an average and smaller value then the magnitude of director distortion at the midplane of the sample, as described by Boudreau et al. (Ref. 7).

A $20 \mathrm{~mm}$ long, $2.8 \mathrm{~mm}$ wide, $50 \mu \mathrm{m}$ deep channel was fabricated using two glass $(n=1.52)$ microscope slides, bonded together by a thermally annealing plastic (Parafilm) to define the channel walls (Fig. 3). Homogeneous surface treatment (AL 1254) was spin coated to a depth of $\approx 100 \mathrm{~nm}$ and rubbed using a velvet cloth at an angle of $\phi_{0}=45^{\circ}$ to the $x$ axis. The flow cell is connected to a Perkin Elmer syringe driver via $0.8 \mathrm{~mm}$ diameter stainless steel tubing (at the cell) and polypropylene hose, with the cell ends sealed using UVcuring glue.

The liquid crystal cell was filled with $5 \mathrm{CB}$ via the stainless steel tubing with a $250 \mu \mathrm{l}$ syringe and left to reach a uniformly aligned, nonflowing homogeneous state. The sample was then placed at the convergence point of the incoming beam and the conoscope aligned to produce an interference figure focused at the charge-coupled device (CCD).

The volumetric flow rate of the syringe driver was increased in steps of $5 \mu \mathrm{l} / \mathrm{h}$ over the range of $0-65 \mu \mathrm{l} / \mathrm{h}$. Images of the interference figure were captured after each increment, allowing time for the system to reach a stable state. In order to accurately measure the angle of conoscopic interference figure rotation, computer code was written to track the mirror plane of the interference figure. Minimising the difference between the two halves of the figure for a straight line at a given angle to the figure's $x$ axis allows for

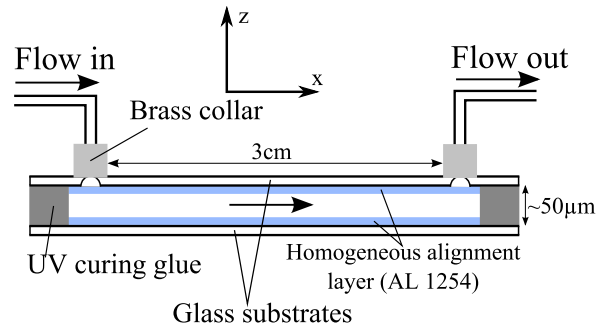

FIG. 3. (Color online) Schematic diagram of pressure driven flow cell designed and constructed.

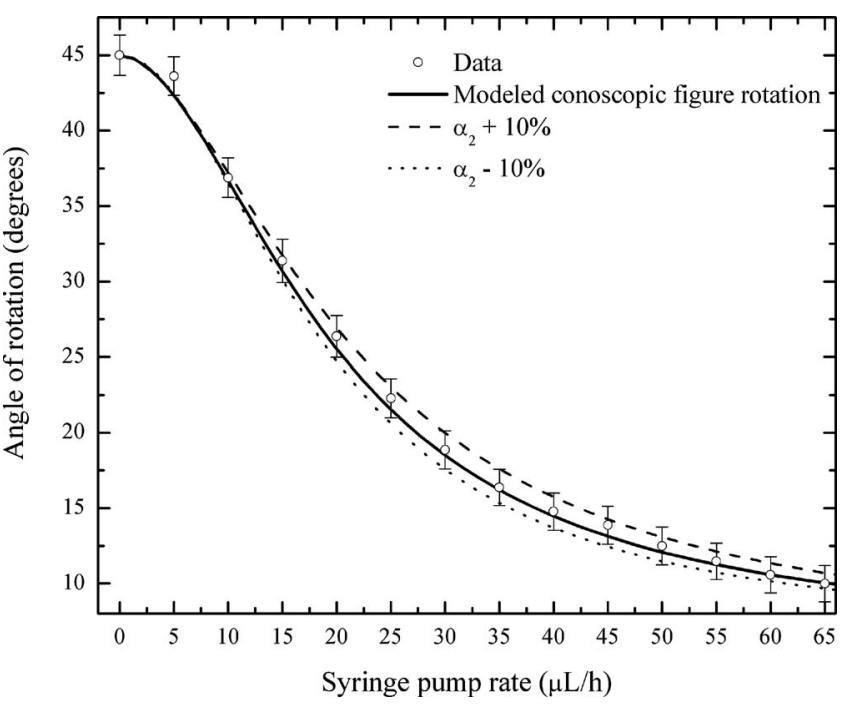

FIG. 4. Measured (symbols) and modeled (solid line) angle of conoscopic interference figure rotation for $5 \mathrm{CB}$ aligned homogeneously at $\phi_{0}=45^{\circ}$ to the direction of pressure driven flow. Dashed and dotted lines show modeled conoscopic figure rotation for $\pm 10 \%$ variations in $\alpha_{2}$.

accurate determination of the figure's rotation as a function of volumetric flow rate.

Figure 4 shows modeled and observed conoscopic interference figure rotation as a function of volumetric flow rate. CCD captures can be seen in Fig. 5, along with modeled conoscopic interference figures for three different volumetric flow rates. As volumetric flow rate increases, the director will begin to rotate out of the flow plane (toward the Leslie angle), creating a torque on the molecules in the azimuthal plane causing an average molecule rotation and conoscopic figure rotation. It is important to note that if there were no $\theta$ rotation of the director out of the flow plane, there would also be no azimuthal $\phi$ rotation. (This could potentially be observed by flowing a negative $\Delta \varepsilon$ liquid crystal while held in planar homogeneous alignment by an external electric field.)
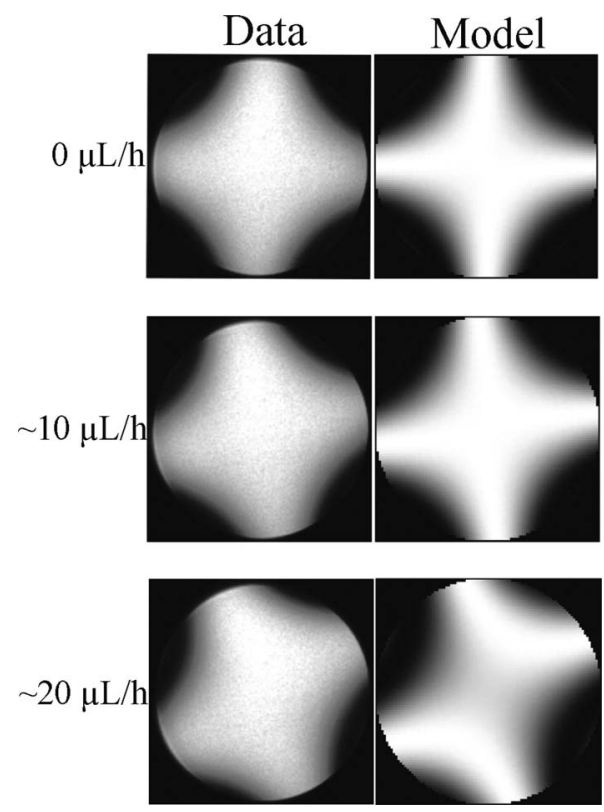

FIG. 5. Captured (left column) and modeled (right column) conoscopic interference figures for $5 \mathrm{CB}$ aligned homogeneously at $\phi_{0}=45^{\circ}$ to the direction of pressure driven flows. 
The Poiseuille flow through a homogeneously aligned cell at $\phi_{0}=45^{\circ}$ to the direction of flow is modeled using Ericksen-Leslie theory with parameters $\left(K_{1}=6.2 \mathrm{pN}, K_{2}=3.9 \mathrm{pN}, K_{3}=8.2 \mathrm{pN}, \alpha_{1}=-0.006 \mathrm{~Pa} \mathrm{~s}\right.$, $\alpha_{2}=-0.0824 \mathrm{~Pa} \mathrm{~s}, \alpha_{3}=-0.0036 \mathrm{~Pa} \mathrm{~s}, \alpha_{4}=-0.0652 \mathrm{~Pa} \mathrm{~s}$, and $\left.\alpha_{5}=-0.0640 \mathrm{~Pa} \mathrm{~s}\right)$. Director profiles for given volumetric flow rates are used to produce theoretical conoscopic interference figures through uniaxial optics code.

It is clear from Fig. 4 that the modeled and experimental data for conoscopic interference figure rotation show excellent agreement. Varying the value of $\alpha_{2}$ (shown by the modeled dashed and dotted lines in Fig. 4) shows an approximate sensitivity of $\pm 10 \%$ to this parameter. It is expected that as the initial alignment angle increases to $90^{\circ}$ (normal to flow direction) a hydrodynamic analog of the Freedericksz transition will be seen as has been previously shown for shear flow. ${ }^{6}$

Translational distortion of the conoscopic figure observed in shear flow experiments ${ }^{7,6}$ is not present under Poiseuille flow regimes due to the symmetry of the velocity distribution about $d / 2$. As discussed earlier, the conoscopic interference figure is an averaging through the depth of the entire cell, and such a symmetric velocity distribution results in a splayed state and an average distortion out of the shear plane of $\bar{\theta}=0$.

We have shown excellent agreement between model and data for the conoscopic figure rotation of a homogeneous liquid crystal cell aligned at $\phi_{0}=45^{\circ}$ to the direction of pres- sure driven flow. Average azimuthal director angle is shown to distort through $35^{\circ}$ (from $45^{\circ}$ to $10^{\circ}$ ) in a nonlinear fashion. Excellent agreement between data and theory confirms the viscosity coefficients of 5CB.

The authors would like to thank the EPSRC and HP for funding this work, along with Sharon Jewell for informative discussion and Nick Cole for technical assistance.

${ }^{1}$ S. Chono, T. Tsuji, and M. M.Denn, J. Non-Newtonian Fluid Mech. 79, 515 (1998).

${ }^{2}$ J. Feng and L. G. Leal, Phys. Fluids 11, 2821 (1999).

${ }^{3}$ J. Fukuda, H. Stark, M. Yoneya, and H. Yokoyama, Phys. Rev. E 69, 041706 (2004).

${ }^{4}$ T. Borzsonyi, A. Buka, A. P. Krekhov, and L. Kramer, Phys. Rev. E 58, 7419 (1998).

${ }^{5}$ J. Quintans Carou, B. R. Duffy, N. J. Mottram, and S. K. Wilson, Phys. Fluids 18, 027105 (2006).

${ }^{6}$ P. Pieranski and E. Guyon, Solid State Commun. 13, 435 (1973).

${ }^{7}$ D. M. Boudreau, H. H. Winter, C. P. Lillya, and R. S. Stein, Rheol. Acta 38, 503 (1999).

${ }^{8}$ I. S. Nasibullayev, O. S. Tarasov, A. P. Krekhov, and L. Kramer, Phys. Rev. E 72, 051706 (2005).

${ }^{9}$ F. M. Leslie, Arch. Ration. Mech. Anal. 28, 265 (1968).

${ }^{10}$ J. L. Ericksen, Mol. Cryst. Liq. Cryst. 7, 153 (1969).

${ }^{11}$ O. J. Parodi, J. Phys. (Paris) 31, 581 (1970).

${ }^{12}$ R. G. Larson and D. W. Mead, Liq. Cryst. 15, 151 (1993).

${ }^{13}$ V. V. Belyaev, Usp. Khim. 44, 255 (2001).

${ }^{14} \mathrm{M}$. Born and E. Wolf, Principles of Optics, 4th ed. (Pergamon, Oxford, 1969).

${ }^{15}$ L. Parry-Jones, E. Kriezis, and S. Elston, Jpn. J. Appl. Phys., Part 1 41, L1485 (2002). 